\title{
Quelques aspects des écoulements bicouches quasi-horizontaux et de leur calcul
}

\author{
PAR
}

\author{
J.-M. Parot \\ Laboratoire National d'Hydraulique, Chatou
}

Nous présentons ici une étude des écoulements quasi horizontaux à deux couches. Nous avons mis en évidence, ou retrouvé certaines propriétés physiques, en étudiant les équations de tels systèmes du point de vue de la propagation et de la stabilité de leurs solutions. Puis, en nous aidant de ces résultats, nous avons mis au point un modèle mathématique qui s'est montré apte à reproduire des phénomènes réels.

\section{Hypothèses et établissement des solutions}

Dans l'espace rapporté aux coordonnées $x, y, z$ et $t$, on considère un système fluide de température variable; il est limité par le fond et une surface libre de cote $Z,(x, y, t)$.

On fait les hypothèses suivantes:

- incompressibilité : les variations de la masse volumique sont suffisamment faibles pour qu'on puisse les négliger dans l'équation de conservation de la masse : le mouvement est à divergence nulle;

- existence d'une diffusion turbulente de la température et de la quantité de mouvement;

- quasi-horizontalité : les composantes de la vitesse et la température varient beaucoup plus lentement dans le plan horizontal que sur la verticale, ceci implique un fond à pente faible;

- on peut négliger les actions de la viscosité et de la conduction dans le sens horizontal;
- le nombre de Froude de l'écoulement n'est pas trop grand (au plus de l'ordre de 1);

- une surface $Z_{1}(x, y, t)$, appelée interface, sépare le fluide en deux zones appelées couches, et dans le calcul du terme de pression on peut considérer la masse volumique comme constante dans chaque couche.

Avec ces hypothèses, on fait la moyenne sur les fluctuations turbulentes des équations tridimensionnelles en densité variable qui régissent le mouvement: conservation de la masse, de la chaleur, et de la quantité de mouvement (Navier-Stokes); puis on les intègre sur la verticale dans chaque couche; l'intégration sur la verticale fait apparaître deux tenseurs de dispersion dans chaque couche (quantité de mouvement et chaleur); cette dispersion est due aux hétérogénéités de vitesse sur la verticale. C'est Taylor [1] qui, le premier, a montré que les hétérogénéités de vitesse dans le sens de l'intégration peuvent être simulées par une diffusion dans le sens de l'écoulement. Cette approche nous donne, en l'absence de mélange entre couches, les équations (S) présentées dans la communication [2], page 3 .

Ce sont elles que nous allons maintenant traiter par diverses méthodes mathématiques.

\section{Méthodes des petites perturbations et stabilité}

Nous nous bornons au cas unidimensionnel et nous supposons la différence des masses volumiques $\Delta \rho$ petite devant la masse volumique $\rho$; les mouvements de la surface sont alors très petits devant ceux de linterface; 
il est possible de remplacer la surface libre par un plafond rigide sans modifier sensiblement les mouvements de l'interface. C'est ce qu'on appelle l'hypothèse du plafond. Les équations ( $S$ ) obtenues au paragraphe précédent se réduisent alors à deux équations de masse et quantité de mouvement en $\Delta \mathrm{H}$, différence des tirants d'eau, et $\Delta U$, différence des débits. Ce système en $\vec{R}(x, t)=(\Delta H, \Delta U)$ possède une solution $\overrightarrow{R_{0}}(x, t)$. Appelons $\vec{r}(x, t)$ une petite variation de $\overrightarrow{R_{0}} \cdot \overrightarrow{R_{0}}+\vec{r}$ est solution du système si $\vec{r}$ vérifie un certain système linéaire $(s)$ d'équations aux dérivées partielles. Si l'on remplace $\vec{r}$ par une de ses composantes de Fourier $\overrightarrow{r_{0}} e^{i k(x-c t)}$, où $k$ est réel positif, le système $(s)$ devient un système linéaire homogène classique qui n'admet de solution que si $c$ et $k$ vérifient la relation de dispersion. La composante de nombre d'onde $k$ est dite stable si son module est borné, c'est-à-dire si $c$ a une partie imaginaire négative ou nulle. Par la relation de dispersion on trouve alors les nombres d'onde stables et les nombres d'onde instables.

La validité de la méthode a été confirmée par ses bons résultats qualitatifs pour divers écoulements monocouches à surface libre.

Son application aux écoulements bicouches donne les résultats suivants :

- En l'absence de dispersion par hétérogénéité de vitesse, l'écoulement uniforme sans frottement devient instable lorsque la différence de vitesse $\Delta u$ devient supérieure à $\sqrt{\varepsilon g H}(\varepsilon=\Delta \rho / \rho, H$ est le tirant d'eau total). On retrouve ici une instabilité du type Kelvin-Helmoltz, mais mettant en jeu l'ensemble des deux couches; ce qui est normal puisque nos équations ne sont pas autre chose que les équations de Navier-Stokes intégrées sur la verticale.

- En présence de dispersion par hétérogénéité de vitesse, l'écoulement uniforme sans frottement devient instable lorsque :

avec :

$$
F_{1}^{2}+F_{2}^{2}>1
$$

$$
F_{i}=u_{i} / \sqrt{\varepsilon g H_{i}}
$$

$u_{i}$ : vitesse de la couche $i$,

$H_{i}$ : épaisseur de la couche $i$.

Les deux critères d'instabilité que nous venons d'écrire correspondent, nous le verrons au paragraphe suivant, à des situations particulières de la propagation interne.

Enfin, on montre par le calcul, dans des cas particuliers, que l'on peut stabiliser l'écoulement en introduisant un transfert de masse à l'interface.

Le calcul a été effectué en particulier dans le cas suivant: la couche inférieure est au repos, la couche supérieure, seule 'en mouvement, a une vitesse $u_{2}$ faiblement supérieure à la vitesse critique $\sqrt{\varepsilon g H_{2 .}}$. On admet un entraînement à la vitesse $w_{c}=E u_{2}$ de la couche inférieure dans la couche supérieure. On trouve que toute perturbation de nombre d'onde plus petit que $k_{0}$ est stable si :

$$
E>C\left(k_{0}\right)\left(1-F_{2}^{-2}\right)^{2}
$$

Si l'on remarque que $F_{2}{ }^{-2}$ peut être considéré comme un nombre de Richardson $R_{i}$, l'égalité dans (1) définit une courbe $E\left(R_{i}\right)$ tout à fait semblable à la courbe expérimentale obtenue par Ellison et Turner dans leur étude de l'entraînement turbulent [3].

Ces calculs de stabilité nous suggèrent donc l'existence d'un lien entre le mélange (entraînement, diffusion) et l'instabilité des ondes longues représentées par les équations du type de Saint-Venant.

L'expérience confirme cette hypothèse. Des essais en canal mettant en jeu deux couches en mouvement dans le même sens ont permis de distinguer deux types d'écoulement interne.

Le nombre de Froude interne $F=\sqrt{F_{1}^{2}+F_{2}^{2}}$, défini au paragraphe suivant, est fondamental : lorsque $F$ est « grand» (au moins de l'ordre de un), les transferts à l'interface, caractérisés par le flux entrant dans la couche inférieure, sont fonction croissante de $F$ (fig. 1). C'est également ce qu'ont trouvé Ellison et Turner dans leur cas particulier. Lorsque $F$ est petit (au plus de l'ordre de deux), les transferts interfaciaux sont nettement plus faibles et semblent dépendre beaucoup de la vitesse relative à l'interface. On peut par exemple trouver une relation $D / \Delta u=f\left(K_{e}\right)$, où $K_{e}=\Delta u^{3} / \varepsilon g \nu$, et où $\nu$ est la viscosité cinématique (fig. 2). Le nombre adimensionnel $K_{e}$ caractérise une interface en milieu infini; naturellement, si l'écoulement au voisinage de l'interface est influencé par les parois, $K_{e}$ n'est plus le seul paramètre dont dépendent les transferts interfaciaux.

D'après ce qui précède on peut donc espérer exprimer les transferts à l'interface comme fonctions des grandeurs moyennes définies pour chaque couche $\left(u_{1}, u_{2}\right.$, $H_{1}, H_{2}$, etc.). Ceci est d'un grand intérêt pour la modélisation mathématique que nous avons entreprise, puisqu'ainsi on peut fermer le problème avec le nombre minimum d'inconnues.

Remarque. - L'intérêt de la méthode des perturbations ne se limite pas aux questions de mélange: voir la thèse citée à la fin de cet article.

\section{3. Étude de la propagation}

Nous voulons maintenant préciser les propriétés de la propagation dans un système bicouche bidimensionnel. Pour cela, considérons les équations $(S)$ privées de leurs termes diffusifs. Elles forment un système d'équations aux dérivées partielles du premier ordre. Nous lui avons appliqué la théorie des bicaractéristiques exposée dans l'ouvrage de Hilbert et Courant [4]. Il s'agit de la généralisation à deux dimensions d'espace de la théorie des caractéristiques bien connue à une dimension. Cette méthode nous donne la forme du cône des bicaractéristiques, c'est-à-dire des «ronds dans l'eau» provoqués par une perturbation ponctuelle du système. Daubert et Graffe l'ont appliquée aux écoulements à une couche [5]. Ils ont montré que la section du cône par le plan $(t=1)$ se compose d'un cercle de rayon $\sqrt{g H}$ ( $H$ tirant d'eau), et du centre du cercle, extrémité du vecteur vitesse $\vec{V}$.

Avec deux couches, les principaux résultats sont les suivants : 


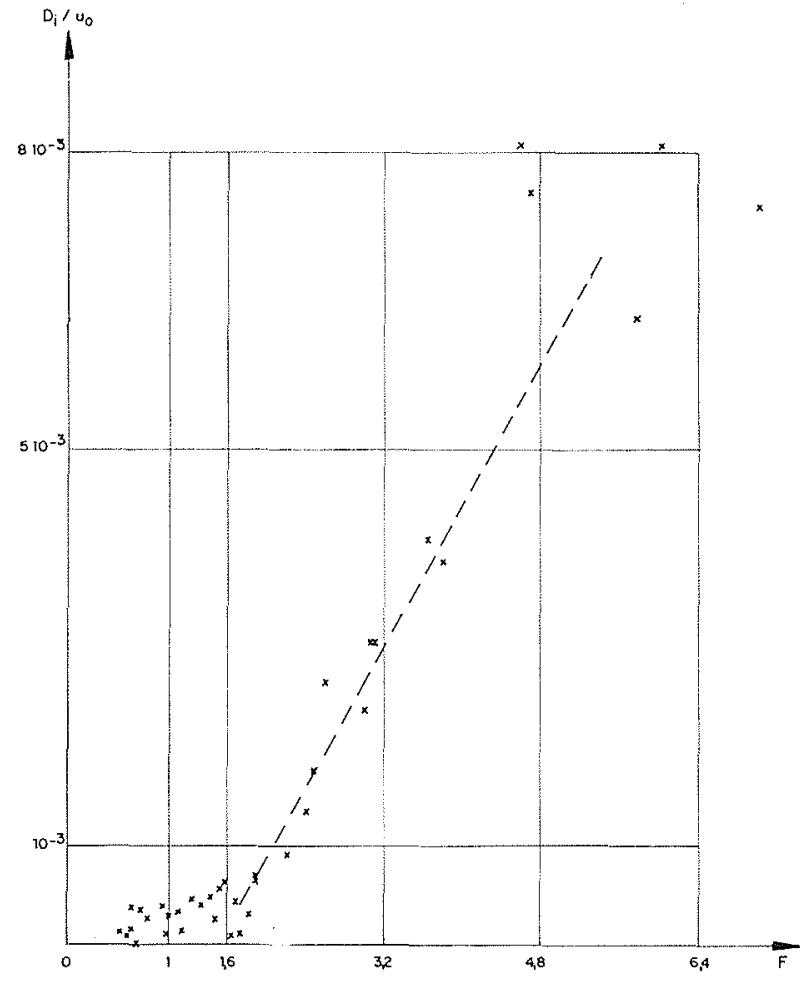

$1 /$
- la section du cône par le plan $(t=1)$ se compose d'une courbe $(\Gamma)$ de classe 4 et de deux points, $M_{1}$ et $M_{2}$, extrémités des vecteurs vitesse $\overrightarrow{V_{1}}$ et $\overrightarrow{V_{2}}$;

- ( $\Gamma$ ) est symétrique par rapport à l'axe $M_{1} M_{2}$;

- à une translation $1 / 2\left(\overrightarrow{V_{1}}+\overrightarrow{V_{2}}\right)$ près, la courbe $(\Gamma)$ ne dépend des vitesses que par $\overrightarrow{\Delta V}=\overrightarrow{V_{1}}-\overrightarrow{V_{2}}$;

- si $\overrightarrow{\Delta V}$ est inférieur à la limite $m$ de stabilité des ondes internes rencontrée au paragraphe précédent, ( $\Gamma)$ se compose de deux courbes fermées d'intersection nulle; la courbe extérieure correspond à la propagation de surface, appelée propagation externe, et la courbe intérieure correspond à la propagation « interne » (voir la fig. 3);

— supposons les vitesses parallèles et $\varepsilon$ très inférieur à 1 . Les célérités dans la direction parallèle à $\overrightarrow{\Delta V}$ sont :

$$
\begin{gathered}
c_{\mathrm{ex}}=\frac{u_{1} H_{1}+u_{22} H_{2}}{H} \pm \sqrt{g H} \\
c_{\mathrm{int}}=\frac{u_{1} H_{2}+u_{2} H_{1}}{H} \pm \frac{\sqrt{H_{1} H_{2}}}{H} \sqrt{\varepsilon g H-\overrightarrow{\Delta V}^{2}}
\end{gathered}
$$

- Lorsque la différence de vitesse tend vers la limite $m$, la courbe «interne» tend vers un segment perpendiculaire à $\overrightarrow{\Delta V}$.

Cette étude nous confirme l'existence de deux propagations, de célérités très différentes lorsque $\varepsilon$ est petit. D'autre part, différence notable avec le monocouche, la forme de la courbe $(\Gamma)$ dépend des vitesses par $\overrightarrow{\Delta V}$; ceci signifie que la convection dans chaque couche interagit avec la propagation à l'interface.

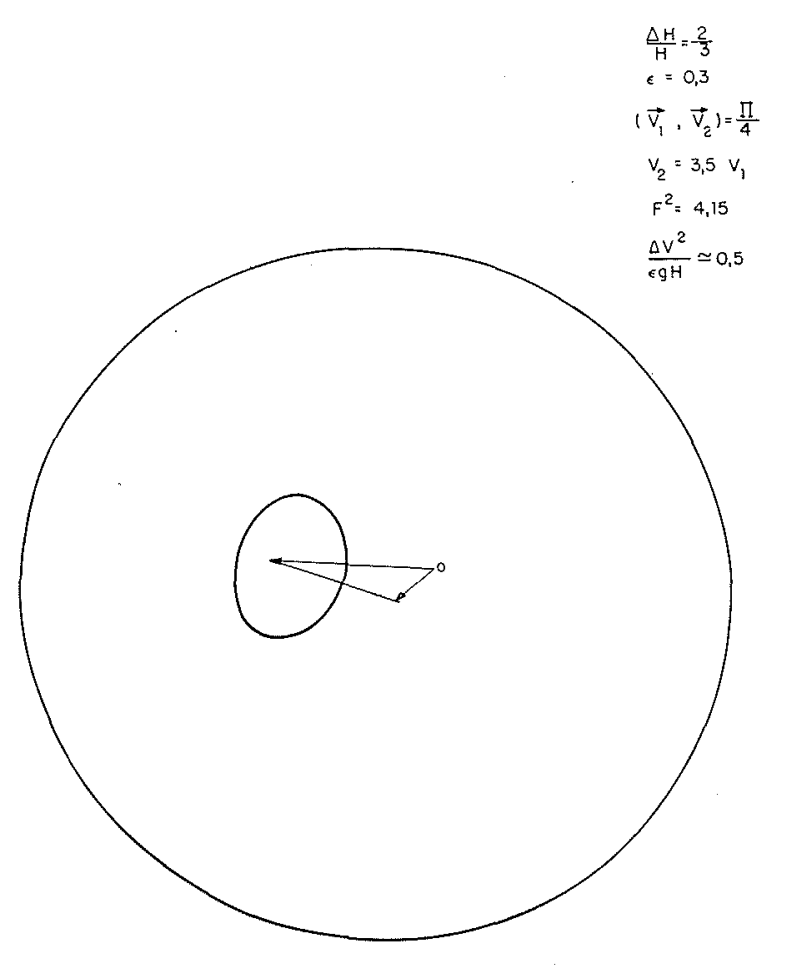

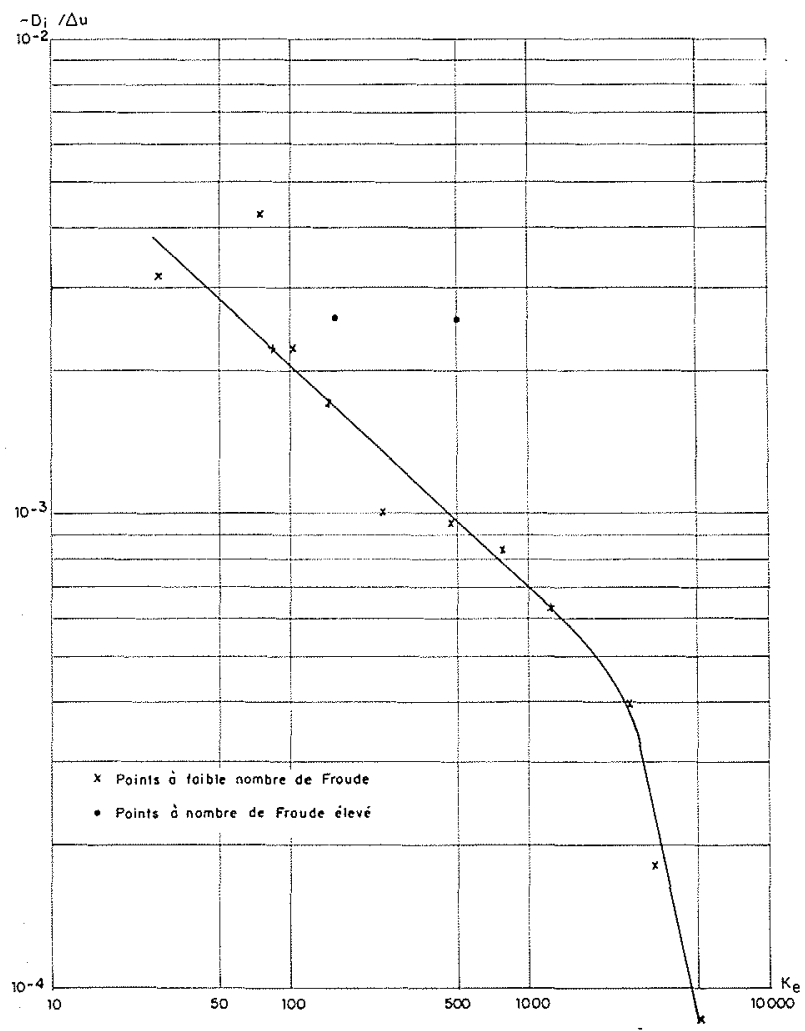

$2 /$ 
Lorsque le cône de propagation possède une bicaractéristique stationnaire, l'écoulement est dit critique. On montre que la condition de criticité interne peut s'écrire :

$$
\left(1-F_{1}{ }^{2}\right)\left(1-F_{2}{ }^{2}\right)=F_{12}{ }^{4}
$$

avec :

$$
F_{1}^{2}=\frac{V_{i}^{2}}{\varepsilon g H_{i}} \quad \text { et } \quad F_{12}^{2}=\frac{\overrightarrow{V_{1} \vec{V}_{2}^{2}}}{\varepsilon g \sqrt{H_{1} H_{2}}}
$$

A une dimension d'espace (2) se réduit à la condition bien connue $F_{1}{ }^{2}+F_{2}{ }^{2}=1$. L'instabilité de l'écoulement dispersif observée au paragraphe 1 apparaît donc quand la diffusion par dispersion longitudinale et la propagation sont antagonistes; en effet, il ne peut y avoir de propagation surcritique vers l'amont, alors que la diffusion est omnidirectionnelle.

De l'expression de la célérité interne en fonction du tirant d'eau de l'une des couches, on peut tirer les conclusions suivantes, en accord avec des observations en nature:

- une onde solitaire, une intumescence, un ressaut interne sont possibles seulement si, $\varepsilon$ étant petit, l'écoulement vérifie la condition:

$$
|\overrightarrow{\Delta V}|<|\Delta H| \sqrt{\varepsilon g / H}
$$

- si la couche inférieure est moins épaisse que la couche supérieure, l'onde est de la forme «directe», c'est-à-dire semblable à une onde de surface libre; sinon elle est de la forme «inversée ».

\section{Le programme de calcul}

On a mis au point et testé un programme de calcul des écoulements bicouches. La principale difficulté du calcul des écoulements bicouches réside dans l'existence de deux célérités de propagation très différentes. En faisant l'hypothèse d'un plafond, nous supprimons la propagation la plus rapide, et cela est très intércssant à une dimension. Mais, en bidimensionnel, l'introduction d'un plafond ne présente aucun avantage de calcul, puisqu'il faut néanmoins résoudre la conservation de la masse sous la forme d'une équation de divergence nulle. Nous avons donc résolu le problème complet, avec les deux propagations.

La méthode repose sur le principe du fractionnement des opérateurs; à chaque opérateur correspond un phénomène physique; nous avons distingué, et traité séparément, diffusion dans chaque couche, convection dans chaque couche, propagation externe et propagation interne. Remarquons que les deux convections et les deux propagations apparaissent distinctement sur la figure 3. Ensuite, nous fractionnons les opérateurs en $x$ et en $y$; les différents opérateurs sont traités successivement avec les conditions aux limites convenables: la diffusion par double balayage, la convection par une méthode de caractéristiques. Le principe du traitement des conditions aux limites est le suivant: les conditions imposées à chaque étape doivent être cohérentes avec la physique de l'opérateur considéré, et, en formant la somme des conditions fractionnées, on doit retrouver les conditions du problème complet.

A cause des deux célérités très différentes, on a été amené à traiter différemment les propagations de surface et d'interface: calcul implicite inconditionnellement stable à la surface (la condition de Courant serait trop contraignante). Par contre, calcul explicite soumis à la condition de Courant à l'interface : n'ayant pas pu mettre en évidence d'invariant pour la propagation interne, on utilise la relation différentielle sur la caractéristique. Dans le calcul de la propagation externe, on traite le terme de cote de l'interface comme le terme de cote du fond, et on utilise pour cela la cote de l'interface au temps précédent. Cette approximation est justifiée car l'échelle de temps des mouvements interfaciaux est beaucoup plus grande que celle des mouvements de la surface.

Certains des fractionnements opérés pouvaient sembler très audacieux, parce que les deux processus séparés interagissent en réalité :

- convection-propagation

- propagation interne-propagation externe;

- propagation en $x$-propagation en $y$;

A l'usage cette façon de procéder s'est montrée justifiée.

\section{Résultats : tests et applications}

Citons les principales solutions littérales, auxquelles les résultats du programme ont été comparés, avec succès.

A une dimension:

- état de repos avec vent:

- états permanents du type coin chaud, coin froid.

- oscillations sinusoïdales de faible amplitude (linéaires) d'un canal:

-- propagation d'une perturbation initiale ponctuelle à la surface, à l'interface.

A deux dimensions:

- propagation d'une perturbation initiale à l'interface.

On a également constaté des résultats qualitativement bons dans les cas suivants:

A une dimension:

- oscillations non linéaires (forte amplitude);

- évolution d'une discontinuité initiale de linterface analogue à la rupture d'un barrage.

A deux dimensions:

- oscillations non linéaires dans un bassin tournant à la suite d'un choc initial:

-- mouvement permanent dans un bassin tournant soumis à un vent constant.

Encouragés par ces résultats satisfaisants, nous avons essayé d’appliquer le programme à deux cas réels attestés par lexpérience: d'ahord le ressaut interne dans le Loch Ness: Thorpe a émis Thypothèse que la thermocline dans le Loch Ness est parenurue par un ressaut interne lorsque le système bicouche est excité par une 

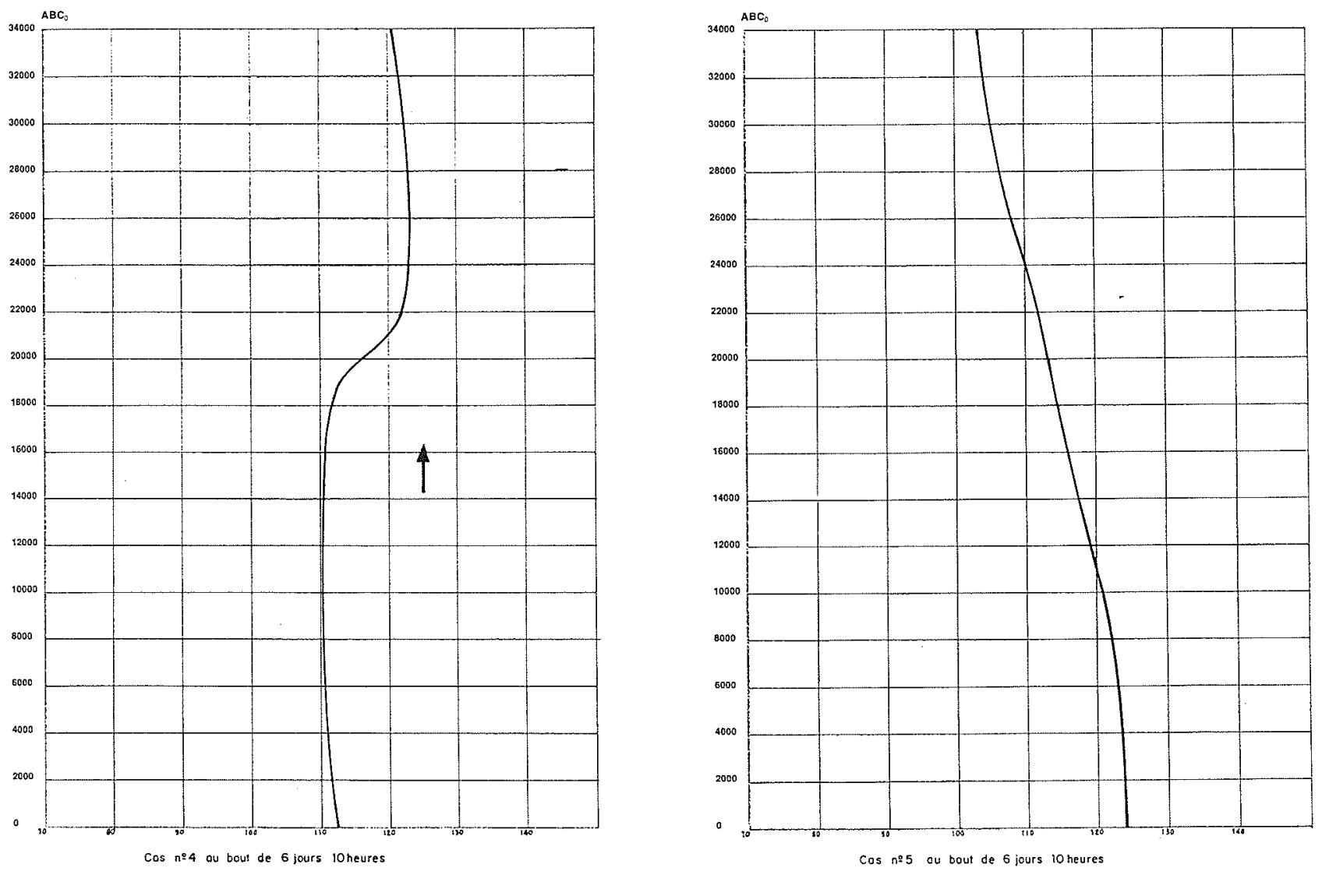

4

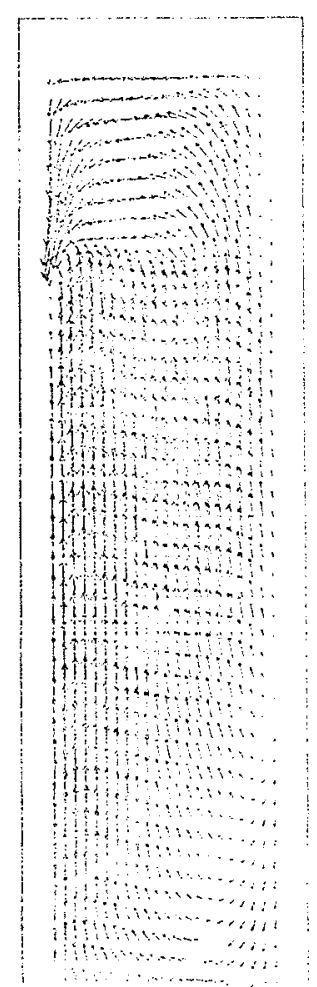


composante du vent de fréquence voisine de sa fréquence propre. Le calcul, effectué avec les valeurs des paramètres physiques données par Thorpe [6] d'après ses mesures, confirme cette hypothèse.

Lorsque les fréquences propres et d'excitation sont suffisamment proches, on voit, au bout de deux à trois périodes, se former une discontinuité mobile (fig. 4). Mais, si elles sont trop différentes (20\% d'écart par exemple), les oscillations du système restent continues (fig. 5).

Le Loch Ness est un cas monodimensionnel; si le bassin est bidimensionnel, la force de Coriolis donne naissance à des mouvements plus complexes: le ressaut formé à la résonance tend à tourner autour du bassin, comme l'ont montré les expériences réalisées à Grenoble par l'équipe de $M$. Chabert-d'Hières. Dans notre modèle de bassin tournant, soumis à une excitation de période propre, on voit effectivement apparaître le genre de phénomène attendu: il se forme une onde raide qui fait le tour du bassin en longeant son bord droit (fig. 6).

Ces exemples montrent l'aptitude du programme à représenter des écoulements réels très non linéaires. On peut maintenant envisager le calcul de phénomènes naturels et, en premier lieu, les mouvements de la thermocline en mer.

\section{Bibliographie}

[1] TAYlor, (Sir Geoffrey). - 1953 a Proc. Roy. Soc. A, $219,186$.

[2] Boulot, Parot, Benque. - Modèles mathématiques bidimensionnels d'écoulements à une ou deux couches. Congrès A.I.R.H., 1975.

[3] Elluison et Turner (J. S.). - Turbulent entrainment in stratified flows. J. of Fluid Mech., 6, 1959.

[4] Courant et Hilbert. - " Methods of mathematical physics ". vol. 2: "Interscience pub.".

[5] Daubert (A.) et Graffe (O.). - Quelques aspects des écoulements presque horizontaux à deux dimensions en plan. La Houille Blanche, 1967, 8.

[6] Thorpe (S. A.). - Near-reasonant forcing in a shallow two-layer fluid: a model for the internal surge in Loch Ness'? J. of Fluid Mech.. 1974, vol. 63, part 3, p. 509.

Cet article résume une thèse présentée à l'Université Paris VI sous le titre: Contribution à l'étude des écoulements bidimensionnels quasi-horizontaux de deux couches de densités différentes. Elle a été préparée dans le cadre d'une bourse de recherche attribuee par l'Ecole Nationale des Ponts et Chaussées, au titre de la Recherche Scientifique et Technique. 